\title{
Urine cell-free DNA is a biomarker for nephroblastomatosis or Wilms tumor in PIK3CA-related overgrowth spectrum (PROS)
}

\author{
Marta Biderman Waberski, MD ${ }^{1,4}$, Marjorie Lindhurst, $\mathrm{PhD}^{1}$, Kim M. Keppler-Noreuil, MD ${ }^{1}$, \\ Julie C. Sapp, ScM ${ }^{1}$, Laura Baker, MGC ${ }^{2}$, Karen W. Gripp, MD ${ }^{2}$, Denise M. Adams, MD ${ }^{3}$ and \\ Leslie G. Biesecker, MD ${ }^{1}$
}

\begin{abstract}
Purpose: We set out to facilitate the molecular diagnosis of patients with PIK3CA-related overgrowth spectrum (PROS), a heterogeneous somatic disorder characterized by variable presentations of segmental overgrowth, vascular malformations, skin lesions, and nephroblastomatosis, rare precursor lesions to Wilms tumor. Molecular diagnosis of PROS is challenging due to its mosaic nature, often requiring invasive biopsies.
\end{abstract}

Methods: Digital droplet polymerase chain reaction (ddPCR) was used to analyze tissues including urine, saliva, buccal cells, and blood, from eight patients with PROS. Further analyses were performed on plasma and urine cell-free DNA (cfDNA).

Results: PIK3CA variants were detected in plasma cfDNA at levels up to $0.5 \%$ in $50 \%$ of tested samples. In addition, high levels of
PIK3CA variants in urine cfDNA correlated with a history of nephroblastomatosis compared with patients without renal involvement $(P<0.05)$.

Conclusion: Digital droplet PCR is a sensitive molecular tool that enables low-level variant detection of PIK3CA in various tissue types, providing an alternative diagnostic method. Furthermore, urine cfDNA is a candidate biomarker for nephroblastomatosis in PROS, which may be useful to refine screening guidelines for tumor risk in these patients.

Genet Med advance online publication 4 January 2018

Key Words: biomarker; cell-free DNA; digital droplet PCR; nephroblastomatosis; PIK3CA

\section{INTRODUCTION}

The PIK3CA-related overgrowth spectrum (PROS) is heterogeneous, including nonsyndromic macrodactyly ${ }^{1}$ to lifethreatening pleiotropic malformations and overgrowth, ${ }^{2}$ and others. ${ }^{3,4}$ The PROS phenotypes are caused by somatic, gainof-function PIK3CA variants. Similar to many mosaic syndromes, the molecular diagnosis of PROS is challenging. The more common causative variants are strongly activating variants that are rarely detectable in peripheral blood. These variants are more readily detected in affected tissue biopsies, which can be challenging to collect because of expense, patient aversion, and challenges in determining which tissues should be selected for testing. Tumor susceptibility in PROS has been recently described in patients with nephroblastomatosis, a precursor lesion to Wilms tumor (WT) ${ }^{5,6}$ A critical challenge in managing these patients is to develop appropriate WT screening methods. This is especially challenging because the variants may not be present in renal tissue in some patients, and therefore WT screening is not appropriate. A method to identify the patients who are at risk would be extremely useful.
To approach the problems of molecular diagnosis of PROS and WT susceptibility, we analyzed DNA from various tissues from patients with PROS to determine if we could detect the variant in samples other than affected tissue biopsies, which is our standard approach. We piloted variant detection in a series of samples from a group of eight patients. These samples included (i) DNA from an affected skin biopsy, (ii) peripheral blood, (iii) circulating cell-free DNC (cfDNA), (iv) urine cellular DNA, (v) urine cfDNA, (vi) saliva, and (vii) buccal swab. To maximize the sensitivity, we used digital droplet PCR (ddPCR), a robust analytic technique that can identify mosaic variants as low as $0.001 \% .^{7}$ We evaluated which samples yielded useful ddPCR results and correlated those findings with clinical manifestations.

\section{MATERIALS AND METHODS}

This study was approved by the National Human Genome Research Institute's institutional review board. Upon consent, the study was initiated with a broad survey of samples from eight affected patients (whole blood, urine, saliva, and cheek swab). All were initially diagnosed with a PIK3CA variant using DNA from an affected tissue biopsy. They had either

\footnotetext{
${ }^{1}$ Medical Genomics and Metabolic Genetics Branch, National Human Genome Research Institute, National Institutes of Health, Bethesda, Maryland, USA; ${ }^{2}$ Division Genetics, Alfred I. duPont Hospital for Children, Wilmington, Delaware, USA; ${ }^{3}$ Division of Hematology/Oncology, Vascular Anomaly Center, Boston Children's Hospital, Boston, Massachusetts, USA. Correspondence: Marta Biderman Waberski (martabiderman@gmail.com)

${ }^{4}$ Current affiliation: Pediatric Specialists of Virginia (Inova Fairfax Community Hospital and Children's National Medical Center), Fairfax, Virginia, USA

Submitted 16 August 2017; accepted 6 November 2017; advance online publication 4 January 2018. doi:10.1038/gim.2017.228
} 


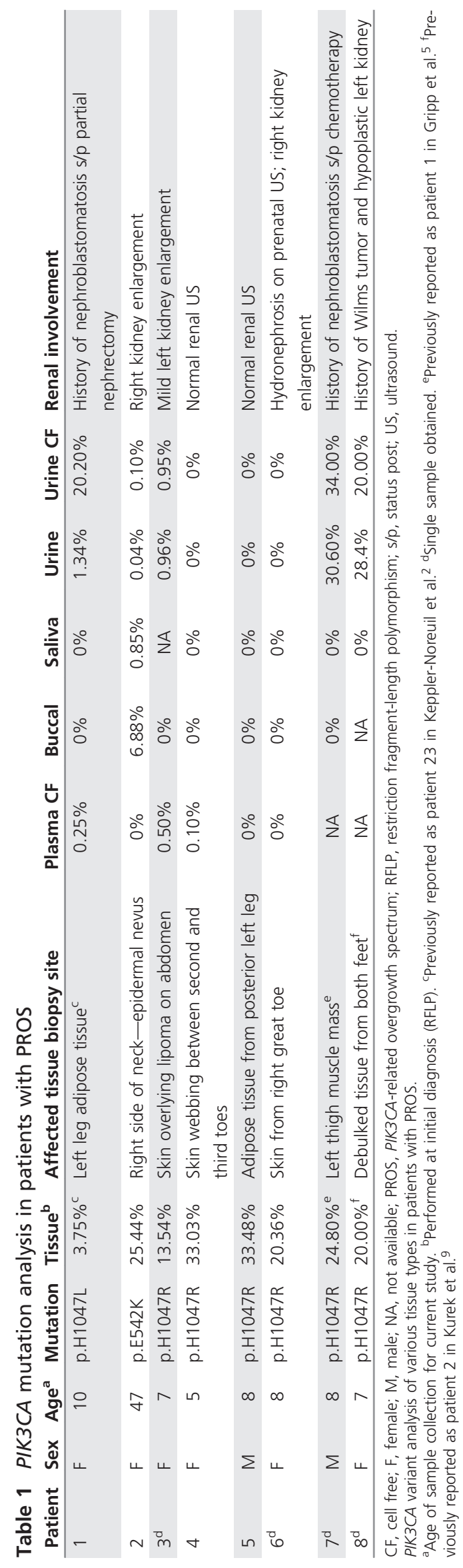

c.3140A > G; p.(His1047Arg), c.3140A > T; p.(His1047Leu), or c.1624G >A; p.(Glu542Lys) (reference NM_003491.3). Genomic DNA was extracted from urine (Urine DNA Isolation Kit; Norgen Biotek, Thorold, ON, Canada), saliva (Gentra PureGene Blood Kit; Qiagen, Germantown, MD), cheek swab (Gentra Puregene Buccal Cell Kit; Qiagen), and whole blood (Gentra PureGene Blood Kit; Qiagen). Urine and blood samples were prepared for cfDNA extraction. Three milliliters of each was centrifuged at $3,000 \mathrm{~g}$ for $10 \mathrm{~min}$ at $4{ }^{\circ} \mathrm{C}$, and resulting supernatant was subject to additional centrifugation at $3,000 \mathrm{~g}$ for $10 \mathrm{~min}$ at $4{ }^{\circ} \mathrm{C}$. These samples were stored at $-80^{\circ} \mathrm{C}$. Cell-free DNA extraction was performed using the QIAmp Circulating Nucleic Acid Kit (Qiagen).

Extracted DNA was quantified using Qubit fluorometry (Thermo Fisher Scientific, Waltham, MA, USA). Each ddPCR assay included triplicates of each sample and we limited the amount of DNA per well to $50 \mathrm{ng}$, or in cases of lower yield, the maximal amount of DNA obtained from each preparation. Each DNA sample was mixed with a primer pair and two TaqMan probes, FAM (mutant sequence) and HEX (wild-type sequence). Following droplet generation (QX200 Droplet Digital PCR system; Biorad, Hercules, CA) and subsequent PCR, samples were analyzed with a fluorescent droplet reader (Biorad), as described. ${ }^{8}$ Negative controls included DNA extracted from similar tissue types, from patients with other variants. We used Quantasoft analysis software (Biorad) to analyze the ddPCR data, and data are expressed as a percentage of mutant droplets to the sum of mutant + wild-type droplets for each sample. Statistical significance was calculated using the unpaired $t$-test, both logtransformed and nontransformed (with zero values set to $0.1 \%$, the lowest nonzero value we detected in this study). Samples were tested together and on two separate time points (unless otherwise specified). Tissues are reported as positive for the variant, if detected on both occasions.

\section{Digital droplet PCR performance}

\section{RESULTS}

The performance of ddPCR was assessed for each probe, through serial dilutions of mutant template with constant wild-type DNA. A positive, linear relationship was observed between mutant DNA, and the concentration determined by ddPCR for each PIK3CA variant (data not shown). In accordance with the rule of threes, we set our threshold as $>3$ positive droplets over the background of any wild-type, false-positive droplets.

\section{Variant detection using ddPCR in various tissues}

Analysis of circulating plasma cfDNA was positive in $3 / 6$ patients, at an average level of $\leq 0.5 \%$ (Table 1 ). The variant was detected in the saliva in $1 / 7$ of tested patients at an average level of $0.85 \%$. This same patient also had the variant present in buccal cells obtained from a clinically affected cheek (6.88\% average variant level). No variants were detected in leukocytes in any of the tested patients (0/6). DNA extracted from urine cellular DNA was positive for the 

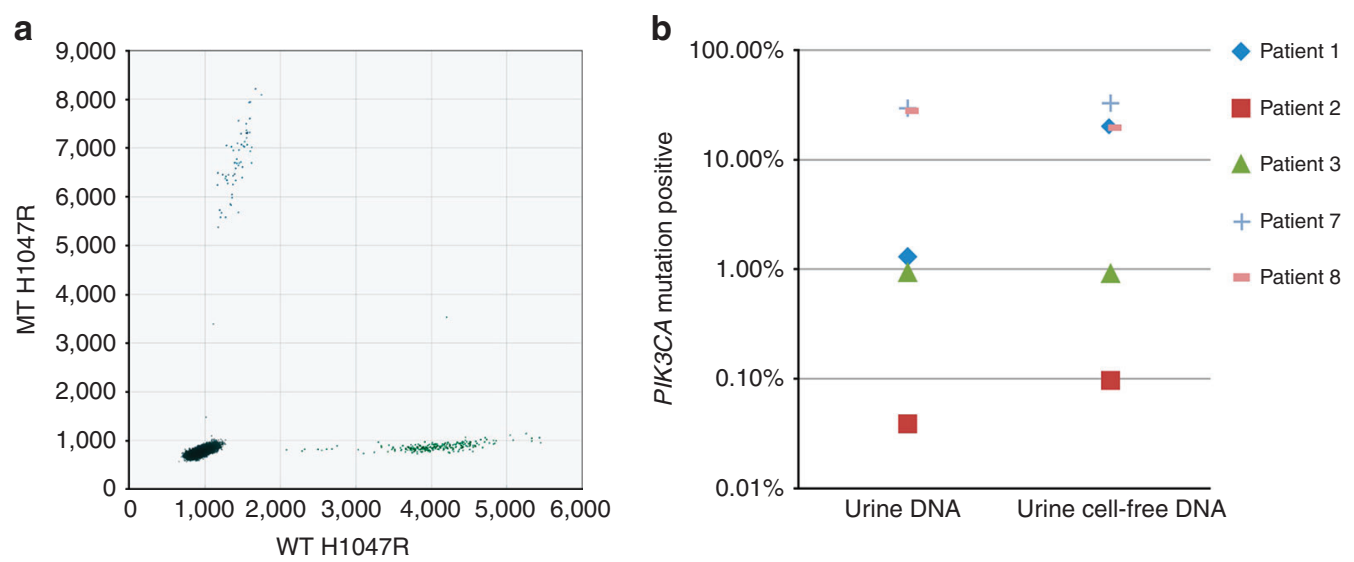

Figure 1 Urine cell-free DNA analysis in PROS patients. (a) Digital droplet polymerase chain reaction results from PIK3CA variant analysis in mutant versus wild-type urine cell-free DNA from patient 8 (p.H1047R) using 2D plot. MT, mutant; WT, wild-type. (b) PIK3CA variant isolated from urine cellular DNA and urine cell-free DNA in PIK3CA-related overgrowth spectrum patients.

PIK3CA variant in 5/8 patients, and these same patients also were variant-positive in their urine cfDNA (Figure 1a). Patients with a history of nephroblastomatosis had markedly increased variant levels in their urine cfDNA at levels of $20 \%$, $35 \%$, and $20 \%$ compared with the other patients with levels of $<1 \%$ (Figure 1b). This difference was statistically significant with untransformed values $(P=0.0004)$ and log-transformed values $(P=0.0002)$. However, only two of these patients had significantly elevated levels of mutant DNA isolated from the cellular urine component, both had a history of renal involvement $(P=0.0001)$. All three patients previously underwent treatment due to the risk of nephroblastomatosis as a precursor lesion for WT.

\section{Renal involvement in patients with PIK3CA variants}

Based on the wide range of variant levels in plasma and urine cfDNA samples, we further explored the histories of these patients. Six patients were clinically evaluated and examined at the National Institutes of Health (Supplementary Table S1 online). No clinical correlation was found for patients with variant-positive plasma cfDNA. One patient with high urine cfDNA variant levels had a history of nephroblastomatosis; therefore, we actively recruited patients 7 and 8 , who had a similar history of renal cell involvement, previously reported by Gripp et al. $^{5}$ and Kurek et al., ${ }^{9}$ respectively. Following routine renal ultrasound imaging, patient 1 developed bilateral renal lesions by age 2, patient 7 had evidence of unilateral lesions at 9 months, whereas patient 8 had bilateral renal lesions by 18 months of age. Initial biopsy of the renal lesions in patient 7 showed nephrogenic rests concerning for WT. Similarly, patient 8 underwent biopsy of a lesion on the left due to initial suspicion of nephrogenic rests on magnetic resonance imaging, confirmed by pathology to be WT. All three patients underwent chemotherapy. Patient 1 had subsequent bilateral nephrectomies, also confirming presence of nephrogenic rests. The lesions remained stable in patient 7 and are currently monitored radiographically. Following initiation of chemotherapy in patient 8 , the left renal lesion increased in size and she subsequently underwent unilateral nephrectomy. The lesions on the right side are stable.

\section{DISCUSSION}

We present a proof-of-concept study that demonstrates the potential utility of samples other than affected tissue biopsies for molecular diagnosis of PROS, using ddPCR. The sensitivity of ddPCR allows variant detection at levels as low as $0.001 \%{ }^{7}$ The sensitivity of ddPCR allowed us to detect low variant levels from plasma cfDNA in $50 \%$ of the patients who were tested, with levels up to $0.5 \%$. PIK3CA variants in these patients were not present in leukocyte-derived DNA; however, the presence of variants in the circulating plasma may signify DNA release from apoptotic cells, from regular cell turnover. We confirm that testing of easily accessible tissues from grossly affected areas leads to an increased variant yield, as seen in saliva and buccal cell samples from patient 2 . Analysis of plasma cfDNA in patients with mosaicism may represent an alternative method of diagnosis in this population; however, further study is needed to validate this approach.

We found high levels of PIK3CA variants in urine cfDNA, and noted that this was correlated with a history of nephroblastomatosis or WT compared with patients without known renal involvement $(P=0.0004)$. Urine cfDNA comprises both transrenal cfDNA and DNA from renal epithelial cell shedding. ${ }^{10}$ Transrenal cfDNA comprises 150 200 bp DNA fragments that are filtered from plasma and likely does not contribute a significant portion to the overall quantity of measureable urine cfDNA. ${ }^{11}$ Despite a similar epithelial origin of DNA from cellular urine isolates, only two of the three patients with nephroblastomatosis had significant amounts of the variant in their sediment. This may represent the PCR inhibitor effect of urea and presence of alternate sources of DNA, including lymphocytes, bacteria, viruses, and parasites. ${ }^{11,12}$ We note that patients 2 and 3 also had measurable variant levels in their urine; however, these were much lower than patients with nephroblastomatosis. We 
hypothesize that it is necessary to have the PIK3CA-activating variant in renal tissue at significant levels for a patient to be at risk for WT and that analysis of urine cfDNA has the potential to identify susceptible patients. Given the size of this study, we cannot exclude that we have identified a spurious association of urine cfDNA and kidney tumors, and larger studies are needed.

Molecular biomarkers from urine cfDNA have been shown to aid diagnosis and disease monitoring for several conditions. Urine cfDNA can facilitate early detection/screening for disease recurrence in bladder cancer, clear-cell renal cell carcinoma, and prostate cancer. ${ }^{13}$ Urinary biomarkers have been US Food and Drug Administration-approved for early cancer detection in prostate and bladder cancers. ${ }^{14}$ Further applications include transplant rejection monitoring and prenatal fetal DNA detection in maternal urine. ${ }^{15,16}$ The sensitivity of ddPCR allows these applications and enhanced diagnosis in genetic disorders, such as Pallister-Killian syndrome and Sturge-Weber syndrome. ${ }^{17,18}$

There are several limitations to this study. The first is that it comprised a relatively small number of patients, with samples obtained at limited time points. The PIK3CA variants represented by this cohort were limited to common PROS variants, ${ }^{2,19}$ and since ddPCR requires predetermined variants, its use is not practical for scanning uncommon variants. Instead, ddPCR may be useful as an initial, less invasive screen for common variants, followed by biopsy with standard variant scanning for those negative by ddPCR. Furthermore, the three patients with nephroblastomatosis and/or WT underwent treatment for renal malignancy and it is possible that the urine cfDNA levels are a consequence of that treatment, rather than the nephroblastomatosis or WT itself. However, we do note that the opposite is seen in other forms of renal cancer where cfDNA levels are decreased posttreatment, and can be used as a monitoring tool. ${ }^{13}$ In addition, we also note that DNA quantity and quality differ among tissue types and our experience highlights the importance of comparing similar tissues when using ddPCR.

We conclude that urine cfDNA analysis using ddPCR is a candidate biomarker for renal involvement in PROS. The apparent correlation of somatic renal variant burden identified through urine cfDNA may allow stratification for WT risk among patients with PROS. Screening guidelines for WT are well established, but it is unclear which patients with PROS warrant such screening. The estimated risk for WT or nephroblastomatosis in PROS may be as high as $1.6 \%{ }^{5}$ however, recommendations for routine renal ultrasound screening are not established due to the imprecision of this estimate. Without further stratification of WT risk for PROS patients, screening of all affected patients would be burdensome and costly. Because PROS is a mosaic disorder, we hypothesize that the only patients who have a risk of nephroblastomatosis and WT are those with a significant burden of renal cells with the PIK3CA-activating variant. In this model, patients with significant renal variant burden have an elevated risk of nephroblastomatosis and cancer whereas patients with variants limited to nonrenal tissues would not have such a risk. The importance of identifying these patients is highlighted by the success in WT treatment, with long-term survival $>90 \%$ for localized disease. ${ }^{20} \mathrm{~A}$ prospective study is needed to explore the variability of urine cfDNA variant levels in patients with PROS and the correlation with renal involvement to further assist in clinical management. Analysis of multiple tissues will assist in understanding the natural history of PROS, properly stratifying patients for WT risk as a pathway to precision medicine in patients with PROS.

\section{SUPPLEMENTARY MATERIAL}

Supplementary material is linked to the online version of the paper at http://www.nature.com/gim

\section{ACKNOWLEDGMENTS}

This work was supported by the Intramural Research Program of the National Human Genome Research Institute grants HG200328 11 and HG200388 03.

\section{DISCLOSURE}

L.G.B. is an uncompensated adviser to Illumina and receives royalties from Genentech. The other authors declare no conflict of interest.

\section{REFERENCES}

1. Rios JJ, Paria N, Burns DK, et al. Somatic gain-of-function mutations in PIK3CA in patients with macrodactyly. Hum Mol Genet. 2013;22:444-451.

2. Keppler-Noreuil KM, Sapp JC, Lindhurst MJ, et al. Clinical delineation and natural history of the PIK3CA-related overgrowth spectrum. Am J Med Genet A. 2014;164A:1713-1733.

3. Lindhurst MJ, Parker VE, Payne F, et al. Mosaic overgrowth with fibroadipose hyperplasia is caused by somatic activating mutations in PIK3CA. Nat Genet. 2012;44:928-933.

4. Jansen LA, Mirzaa GM, Ishak GE, et al. PI3K/AKT pathway mutations cause a spectrum of brain malformations from megalencephaly to focal cortical dysplasia. Brain. 2015;138(pt 6):1613-1628.

5. Gripp KW, Baker L, Kandula V, et al. Nephroblastomatosis or Wilms tumor in a fourth patient with a somatic PIK3CA mutation. Am J Med Genet A. 2016;170:2559-2569.

6. Peterman CM, Fevurly RD, Alomari Al, et al. Sonographic screening for Wilms tumor in children with CLOVES syndrome. Pediatr Blood Cancer. 2017;64:e26684.

7. Day E, Dear P, McCaughan F. Digital PCR strategies in the development and analysis of molecular biomarkers for personalized medicine. Methods 2013;59:101-107

8. Hindson BJ, Ness KD, Masquelier DA, et al. High-throughput droplet digital PCR system for absolute quantitation of DNA copy number. Anal Chem. 2011;83:8604-8610.

9. Kurek KC, Luks VL, Ayturk UM, et al. Somatic mosaic activating mutations in PIK3CA cause CLOVES syndrome. Am J Hum Genet. 2012;90:1108-1115.

10. Hui L, Maron JL, Gahan PB. Other body fluids as non-invasive sources of cell-free DNA/RNA. In: Gahan PB (ed). Circulating Nucleic Acides in Early Diagnosis, Prognosis, and Treatment Monitoring. Dordrecht, The Netherlands: Springer, 2015:295-323.

11. Umansky SR, Tomei L. Transrenal DNA testing progress and perspectives. Expert Rev Mol Diagn. 2006;6:153-163.

12. Huggett JF, Novak T, Garson JA, et al. Differential susceptibility of PCR reactions to inhibitors: an important and unrecognised phenomenon. BMC Res Notes. 2008;1:70.

13. Di Meo A, Bartlett J, Cheng Y, Pasic MD, Yousef GM. Liquid biopsy: a step forward towards precision medicine in urologic malignancies. Mol Cancer. 2017;16:80

14. Salvi S, Gurioli G, De Giorgi U, et al. Cell-free DNA as a diagnostic marker for cancer: current insights. Onco Targets Ther. 2016;9:6549-6559. 
15. Gielis EM, Ledeganck KJ, De Winter BY, et al. Cell-free DNA: an upcoming biomarker in transplantation. Am J Transplant. 2015;15: 2541-2551.

16. Tsui NB, Jiang $P$, Chow KC, et al. High resolution size analysis of fetal DNA in the urine of pregnant women by paired-end massively parallel sequencing. PLoS One. 2012;7:e48319.

17. Uchiyama $Y$, Nakashima M, Watanabe $S$, et al. Ultra-sensitive droplet digital PCR for detecting a low-prevalence somatic GNAQ mutation in Sturge-Weber syndrome. Sci Rep. 2016;6:22985.
18. Fujiki $K$, Shirahige $K$, Kaur $M$, et al. Mosaic ratio quantification of isochromosome $12 p$ in Pallister-Killian syndrome using droplet digital PCR. Mol Genet Genomic Med. 2016;4:257-261.

19. Kuentz P, St-Onge J, Duffourd $Y$, et al. Molecular diagnosis of PIK3CArelated overgrowth spectrum (PROS) in 162 patients and recommendations for genetic testing. Genet Med. 2017;19:989-997.

20. Stefanowicz J, Kosiak M, Romanowicz G, Owczuk R, Adamkiewicz-Drożyńska E, Balcerska A. Glomerular filtration rate and prevalence of chronic kidney disease in Wilms' tumour survivors. Pediatr Nephrol. 2011;26:759-766. 